\title{
A New Attempt to the Classfication of Genus Arabis Growing in Japan, Corea, Saghaline and the Kuriles.
}

By

\begin{abstract}
'Takenoshin, Nakai Rigakuhakushi.
\end{abstract}
Conspectus Specierum.

1.

Cotyledones incumbentes. Radix annua v. biennis. Folia radicalia spathulata. Caulis simplex v. cæspitosus. Flores parvi. Petala $2-3 \mathrm{~mm}$. longi. Siliqua $1-2 \mathrm{~cm}$. longa. Semen ellipsoideum exalatum. ...Stenophragma Thalianum, Celak.

$\begin{array}{lllllllllll}\text { Cotyledones accumbentes. } & \ldots & \ldots & \ldots & \ldots & \ldots & \ldots & \ldots & \ldots & \ldots & 2\end{array}$

2. $\quad\left\{\begin{array}{lcccccccccccc}\text { Semina biserialia. } & \ldots & \ldots & \ldots & \ldots & \ldots & \ldots & \ldots & \ldots & \ldots & \ldots & \ldots & 3 \\ \text { Semina uniserialia. } & \text { Flores albi, lilacini } & \text { v. } & \text { purpurei } & & \ldots & \ldots & 6\end{array}\right.$

Radix annua. Caulis simplex v. perennis. Folia radicalia lyrata,

3. caulina oblanceolata glabra. Racemus terminalis. Siliqua 3 $\mathrm{cm}$. longa $1 \mathrm{~mm}$. lata. Semina alata.

$\begin{array}{llllllllll}\ldots & \ldots & \ldots & \ldots & \ldots & \ldots & \ldots & \ldots & \text { Arabis Kawasakiana, MAKINo. }\end{array}$

$\begin{array}{llllllllllll}\text { Radix biennis } \mathrm{v} \text {. perennis. } & \ldots & \ldots & \ldots & \ldots & \ldots & \ldots & \ldots & \ldots & \ldots & 4\end{array}$

(Siliqua elongata pendula v. divaricato-nutans. Flores lilacini v. purpurei v. albidi. Radix biennis. Caulis patentim hirsutus.

4. Folia caulina subamplexicaulia hispidula. Semina alata.

Siliqua elongata erecta stricta. Flores flavidi v. flavido-albidi. Semina cxalata. Folia caulina sagittata glaberrima. $\ldots \quad 5$

$5\left\{\begin{array}{ccccccccccc}\text { Radix } & \text { biennis. } & \ldots & \ldots & \ldots & \ldots & \ldots & \ldots & \ldots & \text { Arabs glabra, BeRNH. } \\ \text { Radix } & \text { perennis incrassata lignosa } & \text { apice cum rosulis. } & \ldots & \ldots & \ldots \\ \ldots & \ldots & \ldots & \ldots & \ldots & \ldots & \ldots & \ldots & \ldots & \ldots & \text { Arabis columnalis, } \\ \text { NAKAI. }\end{array}\right.$

6. $\left\{\begin{array}{l}\text { Folia caulina basi attenuata sessilia v. petiolata. } \\ \text { Folia caulina basi dilatata amplexicaulia. }\end{array}\right.$ 
- Stolonifera. Folia caulina obovata v. spathulata grosse paucique dentata $\mathbf{v}$. interdum argute dentata. Caulis brevis

7. Racemus elongatus. Petala alba $1 \mathrm{~cm}$. longa.

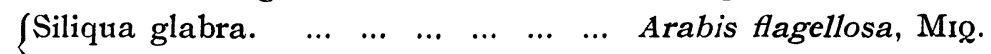
ISiliqua hispida. ... $\ldots$ A. flagellosa.v. lasiocarpa, MAтsum. $\begin{array}{llllllllllllllll}\text { Estolonifera. } & \ldots & \ldots & \ldots & \ldots & \ldots & \ldots & \ldots & \ldots & \ldots & \ldots & \ldots & \ldots & \ldots & 8\end{array}$ (Caulis flaccidus ramosus innovationes axillares agit glaber $\mathbf{v}$. 8. hirsutus. Folia radicalia lyrata, caulina obovata dentata v. sinuata. Petala alba v. lilacina. v. carnea usque $8 \mathrm{~mm}$. longa. Semina marginato-alata. Arabis senanensis, Makino.

$\begin{array}{lllllllll}\text { Caulis innovationes axillares non agit. } & \ldots & \ldots & \ldots & \ldots & \ldots & \ldots & 9\end{array}$

Caulis simplex gracilis. Radix biennis. Planta cum soboles perennis. Folia radicalia rotundata, caulina lanceolata $\mathbf{v}$. elliptica. Racemns laxus. Siliqua 1-1.7 cm. longa. Petala alba v. lilacina usque $5 \mathrm{~mm}$. longa. Semina exalata. $\ldots .$. $\begin{array}{lllllllllllll}\ldots & \ldots & \ldots & \ldots & \ldots & \ldots & \ldots & \ldots & \ldots & \ldots & \ldots & \ldots & \text { Arabis Halleri, } \mathrm{L} .\end{array}$

$\begin{array}{lllllll}\text { Multicaulis et si simplex folia radicalia lyrata. } & \ldots & \ldots & \ldots & \ldots & 10\end{array}$

Radix crassa perennis. Folia radicalia spathulata, caulina lanceolata serrata. Pedicelli erecti. Petala alba 7-9 mm. longa. Siliqua glaberrima. ... $\quad \ldots$ Arabis yokoscensis, Fr. et SAv.

10.

Radix crassa $\nabla$. tenuis perennis. Folia radicalia lyrata, caulina oblanceolata v. linearia integra $v$. serrata. Petala alba 4-5 mm. longa. Siliqua glaberrima 3-6 cm. longa $1 \mathrm{~mm}$. lata. Semina alata.

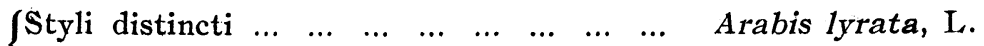

SStyli nulli v. subnulli. ... A. Iyrata v. kamschatika, Fischer.

Planta littoralis cum siliquis strictis crassis dense collocatis. Radix perennis $\mathbf{v}$. biennis. Folia radicalia spathulata $\mathbf{v}$. oblanceolata stellulato-pilosa, caulina ovata v. elliptica v. late ovața. Calyx saccatus. Petala alba 7-10 mm. longa. Semina marginato-alata.

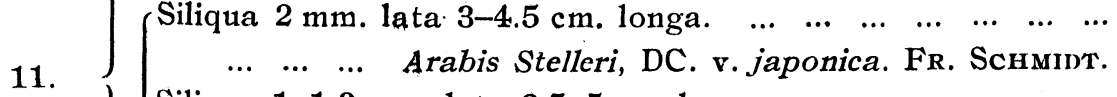
$\begin{array}{llllllll}\text { Siliqua } & 1-1.3 \mathrm{~mm} \text {. lata } 2.5-5 \mathrm{~cm} \text {. longa. } & \ldots & \ldots & \ldots & \ldots & \ldots & \ldots\end{array}$ $\begin{array}{llllll}\ldots & \ldots & \ldots & \ldots & \ldots & \ldots\end{array}$. Stelleri, DC. v. stenocarpa, FR. et SAv. Siliqua circ. 1-1.3 mm. lata $5-7.5 \mathrm{~cm}$. longa. Caulis flexuosus. $\begin{array}{lllllll}\ldots & \ldots & \ldots & \ldots & \ldots & \ldots . . . & \text { Stelleri, DC. v. macrocarpa, NAKAI. }\end{array}$ Flores fere duplo minores quam allia. Siliqua ignota. ... ...

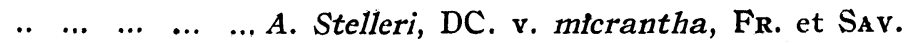

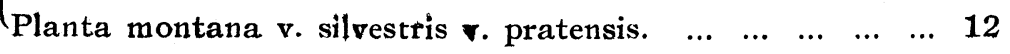


(Planta insulæ Ooryöngto. Caulis erectus simplex v. cæspitosus. Folia caulina præter marginem glaberrima. Petala alba 7-8 $\mathrm{mm}$. longa. Siliqua $1 \mathrm{~mm}$. lata $5-8 \mathrm{~cm}$. longa flexuosa $\mathrm{v}$.

12. secundatim reflexa. Semina marginata.

(Folia caulina ovata. ... $\ldots . . . . .$. Arabis takesimana, NAKAI.

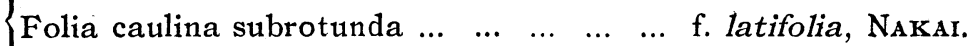
Folia caulina lineari-lanceolata $\ldots . . . .6 . \quad$ f. lancea, NAKAr.

Plantan hondoensis $v$. yesoensis. $\quad \begin{array}{lllllllll} & \ldots & \ldots & \ldots & \ldots & \ldots & \ldots & \ldots & 13\end{array}$

Folia glatca radicalia ovato-oblonga, caulina lanceolato-linearia

13. caulem auriculis acutis amplectentia. Siliqua sæpe torulosæ. Semina marginato-alata. $\ldots . . . . \quad \ldots . .$. Arabis glauca, Borss.

$\begin{array}{lllllllllllllll}\text { Folia viridia. } & \ldots & \ldots & \ldots & \ldots & \ldots & \ldots & \ldots & \ldots & \ldots & \ldots & \ldots & \ldots & \ldots & 14\end{array}$

Caulis simplex siliquis angustis strictis densitus collocatis. Folia radicalia spathulata, caulina ovata $\nabla$. lanceolata omnia stellulata pilosa. Petala alba 5-6 mm. longa. Siliqua 4-5

14. $\left\{\begin{array}{lllllll}\mathrm{cm} \text {. longa } 1 \mathrm{~mm} \text {. lata. Semina exalata. ... } & \ldots & \ldots & \ldots & \ldots & \ldots\end{array}\right.$ $\begin{array}{lllllllllll}\ldots & \ldots & \ldots & \ldots & \ldots & \ldots & \ldots & \ldots & \ldots & \ldots & \text { Arabis nipponica, Borss. }\end{array}$

Caulis sæpe cæspitosus cum siliquis torulosis v. flexuosis v.

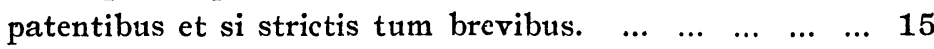

Caulis 3-6 em. altus. Caudex ramosa. Folia radicalia spathulato-linearia, caulina oblongo-lanceolata v. oblongo-ovata

15. semiamplexicaulia, omnia stellulata. Petala $2 \% / 3 \mathrm{~mm}$. longa. Siliqua $11 \mathrm{~mm}$. longa $1 \mathrm{~mm}$. lata patentia. Semina exalata. $\begin{array}{llllllllll}\ldots & \ldots & \ldots & \ldots & \ldots & \ldots & \ldots & \ldots & \ldots & \text { Arabis Tanakana, Makino. }\end{array}$

$\begin{array}{llllllllllllllll}\text { Caulis elatior. } & \ldots & \ldots & \ldots & \ldots & \ldots & \ldots & \ldots & \ldots & \ldots & \ldots & \ldots & \ldots & \ldots & \ldots & 16\end{array}$

Semina immarginata. Pedicelli arcuati. Folia oblongo-spathulata, caulinal anceolato-amplexicaulia, omnia stellulato-pilosa. Siliqua circ. $1.5 \mathrm{~mm}$. lata $4-7.5 \mathrm{~cm}$. longa torulosa. $\quad \ldots \quad \ldots$ $\begin{array}{llllllllll}\ldots & \ldots & \ldots & \ldots & \ldots & \ldots & \ldots & \ldots & \text { A rabis pseudoauriculata, Borss. }\end{array}$

$\begin{array}{lllllllllllllll}\text { Semina alata. } & \ldots & \ldots & \ldots & \ldots & \ldots & \ldots & \ldots & \ldots & \ldots & \ldots & \ldots & \ldots & \ldots & 17\end{array}$

Folia distincte serrata caulina $1.5 \mathrm{~cm}$. haud superantia proxime posita cum ciliis stellulatis crebris. Caudex ramosa. Folia radicalia spathulata serrata. Petala alba 8-9 $\mathrm{mm}$. longa.

17. Siliqua curvata $1.5-2 \mathrm{~mm}$. lata $4-4.5 \mathrm{~cm}$. longa. $\ldots . .$. $\begin{array}{lllllllll}\ldots & \ldots & \ldots & \ldots & \ldots & \ldots & \ldots & \ldots & \text { Arabis serrata, Fr. et SAV. }\end{array}$

Folia caulina indistincte serrata et si distincte serrata $2 \mathrm{~cm}$. $\begin{array}{llllllll}\text { superantia et rarius stellulata. } & \ldots & \ldots & \ldots & \ldots & \ldots & \ldots & 18\end{array}$

(Siliqua fere rectiuscula longe attenuata usque $4 \mathrm{~cm}$. longa. Folia

18. caulina 1-2 cm. longa subglabra $v$. minute stellulata indistincte paucique serrata. $\ldots . .$. Arabis Kishidai, NakaI. 
Siliqua plus minus torulosa $\nabla$. flexuosa v. patentia vulgo quam $\begin{array}{lllllllllllll}4 \mathrm{~cm} \text {. longior. } \ldots & \ldots & \ldots & \ldots & \ldots & \ldots & \ldots & \ldots & \ldots & \ldots & \ldots & 19\end{array}$

19. $\left\{\begin{array}{lllllll}\text { Folia caulina vulgo quam } 2 \mathrm{~cm} \text {. breviora. } \ldots & \ldots & \ldots & \ldots & \ldots & 20\end{array}\right.$

Folia caulina quam $2 \mathrm{~cm}$. longiora, sæpe $3-5 \mathrm{~cm}$. longa. .. 21

(Petala 7-9 mm. longa. Siliqua vulgo 1-1.5 mm. lata. ... ...

20.

Petala 11-12 mm. longa. Siliqua usque $2 \mathrm{~mm}$. lata. $\ldots . . . \quad \ldots$ ...

(Fere semper pauci-multicaulis. Siliqua 4-6.5 $\mathrm{cm}$. longa $1.3-1.5$ $\mathrm{mm}$. lata. Folia caulina oblongo-ovata acuta v. obtusiuscula

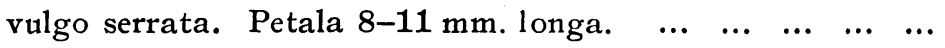
$\begin{array}{llllllllll} & \ldots & \ldots & \ldots & \ldots & \ldots & \ldots & \ldots & \ldots & \text { Arabis Boissieuana, NAKAI. }\end{array}$

Fere semper pauci-multicaulis. Siliqua usque $9 \mathrm{~cm}$. longa 1.5

21. mm. lata. Pedicelli elongati. Petala alba 10-12 mm. longa. Folia caulina magna grosse-serrata. $\begin{array}{lllllll}\ldots & \ldots & \ldots & \ldots & \ldots & \text { Arabis Boissieuana v. shikokiana, NAKAI. }\end{array}$

Caulis fere semper simplex. Folia caulina vulgo undulata v. indistincte remoteque serrata oblonga $v$. clliptica obtusa $v$. acutiuscula. Petala alba $\mathbf{7 - 1 0} \mathrm{mm}$. longa... $\ldots . . . \quad \ldots \quad \ldots$

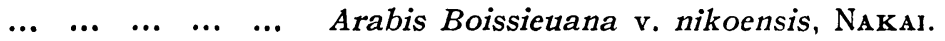

In Corea, there is one more species called Arabis axillaris, Ком. which is likely of a different genus. It is also questionable that Sisymbrium Maximowiczii, Palrb. is verily a species of the genus, though we can not determine their true systematic position by the lack of its ripen fruits. Those two species resemble with each other in their appearance except the former's slightly flattened siliques with short beak and the latter's cylindrical one with long beak. So far as my imagination can go, both belong to an undescribed genus.

Here I have enumerated Stenophragma Thaliana, because it has often been classed under Arabis. As I have stated in the key, Stenophragma has an incumbent cotyledones and the seeds are more roundish than those of Arabis.

Enumeratio specierum.

1) Arabis glabra, (L.) Bernh. Verz. Syst. Erf. (1800) p. 195. Britton and Brown Illus. Fl. II. p. 150. Koidz. Pl. Sachal. Nakah. p. 71.

A. glabra, (L.) Crantz. Hook. Fl. Brit. Ind. I. p. 135 
A. glabra, (L.) Weinm. in Cat. Hort, Dorp. (1810) p. 18. Halacsy Consp. F1. Græc. I. p. 51.

A. perfoliata, Lam. Encycl. I. (1783) p. 219. Sowerby Engl. Bot. I. t. CXIX. Wagn. Deutsche Fl. p. 45 f. 70. Fitch et Smith Illus. Brit. Fl (1y01) f. 56. S. Watson Syn. Fl. I. p. 160. Forbes et Hemsl. Journ. Linn. Soc. XXIII. p. 42. Fran. et Sav. Enum. Pl. Jap. I. p. 34. Borss. in Bull. Herb. Boiss. VII. (1899) p. 785. Kom. Fl. Mansh. II. p. 375. Nakai Fl. Kor. I. p. 53. II. p. 440. Matsum. Ind. P1. Jap. 2. p. 148. Mryabe et Mryake Fl. Sachal. (1915) p. 41.

Turritis glabra, L. Sp. Pl. (1753) p. 636. DC. Prodr. I. p. 142. Ledeb. Fl. Ross. I. p. 116. Косн. Syn. Fl. Germ. et Helv. p. 33. Regel Tent. Fl. Uss. n. 62 et P1. Radd. p. 160. Fr. Schmidt Reis. p. 111. Mre. Prol. Fl. Jap. p. 363. Maxim. Fl. Mongol. p. 44.

T. macrocarpa, Nutr. in TorR. et Gray Fl. North Americ. I. p. 78. Nom. Jap. Hatazao.

Hab.

Corea: Andong (Smith n. 67), Riri (Takenoshin Nakai n. 4798). Monte Chirisan (Takenoshin Nakai n. 49). insula Okto (Takenoshin Nakai n. 251). Monte Namsan (Tomijiro Uchiyama). Pyengyang (Hanjiro Imai). Pede montis Kokyosan in Suigen (Rishoko). Josin (Takenoshin NakaI n. 4796), in littore Ouensan ('Takenoshin NAKaI n. 4797. FaURIE n. 135), prope sepulturam Syou-ouen (FaURIE 11. 131).

Quelpært: inter Taisei et Hokanri (Takenoshin NAKaI n. 4795).

Hondo: in oppido Nobemura prov. Totomi (Kadotaka Hisamatsu). monte Nunobikiyama prov. Settsu (Tomijiro Uchiyama). Hikami in oppido Ouchimura prov. Suwo (Jiuro Nikai n. 244).

Yeso: Sapporo (Kingo Miyabe). Zenibako (Y. Tokubuchi).

Sachalin: ad ripas fluminis Riutoka (Genjr Nakahara).

Distr. Europa, Asia et America bor.

2) Arabis columnalis, NAKAI in Fedde Rep. XIII (1914) p. 271 et Report Veg. m't Chirisan (1915) p.33. n. 209.

Nom. Jap. Inu-hatazao

Hab.

Corea: in Panja montium Chirisan (Takenoshin NaKaI n. 401).

Planta endemica!

3) Arabis pendula, L. Sp. Pl. p. 665. DC. Prodr. I. p. 147. Turcz. Cat. Baic-Dah. n. 116. Less. in. Linnæa IX. p. 153. Ledeb. Fl. Ross. I p. 122. Maxim. Prim. Fl. Amur. p. 44 Fl. Mongol p. 43, MiQ. Prol. Fl. Jap. p. 4. Fran. et SAv, Enum. Pl. Jap. I p. 33. Fr. Schmidt. 
Reis p. 112. RegeL 'Tent. Fl. Uss. p. 21. Pl. Radd. p. 169. Borss. Bull. Herb. Boiss. VII. (1899) p. 786. Kом. Fl. Mansh. II. p. 375. Matsum. in Tokyo Bot. Mag. XIII (1899) p. 59 et Ind. Pl. Jap. II. 2 p. 148. Nakai Fl. Kor. I. p. 53. Koldz Pl. Schal. p. 72. Miyane et Miyake Fl. Sachal. (1915) p. 40.

A. hirsuta, (non Scop.) KoIdz. 1.c.

Nom. Jap. Ezo-hatazao.

Hab.

Sachalin: Toreipachi et Merea (Grjur Nakahara).

Yeso: Sapporo (Kingo Miyabe). Riruran prov. Kushiro (Kingo MiYabe).

Hondo: Usuitoge prov. Shinano (Yoshisada YABE). Nakano prov. Mutsu (Nobctaro Kinashi).

Corea : monte Kum-gang-san (Takenoshin Nakal n. 5447). monte Sai-ka-rei (TAMfzo MoRI n. 234). Kan-gei (Mrlls n. 468). monte Muranbon circa Pyeng-yang (Hanjiro ImaI). inter Kaijyo et Kinsen ('Tomjuro Uchiyama). inter Zuiko et Fu-ju-in (Tomjiro Uchiyama). So-san (Hanjiro Imar), in lacunis interioris regionis (FaUrie n. 122). in vallibus Ouensan (FAURIE $n$. 563). in arenosis Seoul (Faurie $\mathfrak{n}$. 559). in montibus Ouensan (FAURIE n. 121).

Amur : ad stationem Innokentievskaja (V. Komarov. n. 783).

Manchuria: Peuk-nyong prov. Shingkin (Yoshisada. YabE). Senzan (Yoshisada YABE).

Mongolia : Kalatsin (Tatsuzo TorII) planta typica !

China: Utyaisan prov. Shansi (Yoshisada YabE). Pyak-hoa-san prov. Chihli (Yoshisada YABE). Siao-wu-tai-shan 4500 ped. prov. Chihli (Yoshisada YaBe).

Distr. Sibiria, Altai et Rossia.

4) Arabis flagellosa, Mig. Prol. Fl. Jap. (1866-8). p. 4. Fr. et SAv. Enum. Pl. Jap. I. (1875) p. 33. Borss in Bull. Herb. Boiss. VII. (1899) p. 787. Matsum. in Tokyo Bot. Mag. XIII. (1899) p. 59. Ind. Pl. Jap. II. 2. (1912) p. 147. Makino in Somokudzusetsu Rev. Edit. (1912) p. 881. Pl. 725.

Nom. Jap. Suzushiro-so.

Hab.

Shikoku : in Nanogawamura prov. Tosa (K. WAtana BE).

Hondo: Kurashiki prov. Bitchu (Gen-rchi Kordzumi).

var. lasiocarpa, Matsum. 1.c.

Hab.

Kiusiu : Yabakei prov. Bungo (HAMADs). 
Planta endemica!

5) Arabis lyrata, L. Sp. P1. (1753) P. 685. ed. II. (1763) p. 929. DC. Prodr. 1. (1824) p. 146. A GRAY Pl. Jap. p. 307 Syn. Fl. I. (1888) p. 159. Fran. et Say. Enum. Pl. Jap. I. (1875) p. 33. A Gray et S. Wats. Syn. Fl. I. i (1895) p. 159.

Cardamine spathulata, Michx. Fl. Bor.-Americ. 2. (1803) p. 29.

Nom. Jap. Miyama-hatazao.

Hab.

Hondo: monte Togakushiyama (Jinzo Matsumura). Higashiyama circa Aidzu prov. Iwashiro (GEN-ICHI KordzUMI). Usuitoge prov. Shinano (Yoshisada YaBe).

Distr. America bor.

var. kamtschatika, Fischer in litt. fide DC. Prodr. II. (1824) p. 146.

Arabis kamtschatica, (non WiLLd.) Fischer apud Ledeb. Fl. Ross. I. (1842) p. 121.

A. arenosa, (non Scop.) Cham. et Schlecht. in Linnæa I. p. 17.

A. petræa $\delta$. kamtschatica, (FISCHER) REGEL Pl. Radd. I. (1861) p. 167. t. V. f. 5. 6. 7. Matsum. in Tokyo Bot. Mag. XIII. (1899.) p. 59.

A. lyrata, (non L.) Ком. Fl. Mansh. II. (1904) p. 377. NAKaI Fl. Kor. I. p. 54.

A. lyrata var. occidentalis, S. WATs. Syn. Fl. I. (1895) p. 159.

Nom. Jap. Miyama-hatazao.

Differt a typo stigmate sessile.

Hab.

Shikoka : monte Tsurugisan (GEN-ICHI KoIdzUmi).

Hondo: monte Yatsugatake (Yoshisada YABE). monte Shirouma prov. Eitchu (Gen-ichi Koldzumi. Matsuwaka Kishida). Hirasawa prov. Shinano (Jinzo Matsumura). monte Togakushi (Jinzo Matsu. MURA). monte Orengesan prov. Echigo. (MatsuWaka Kishida). monte Shakushi prov. Etchu. (Matsuwaka Kishida).

Yeso: Asahigawa (Hideo Koldzumi). Urup. (Ko'tora Jinbo).

Corea : ad fontes fl. Julu, in valle Tadin-don magna (V. Komarov), Distr. Kanitschatica et America boreali-occid.

6) Arabis Halleri, L. Sp. Pl. ed. II. (1763) p. 929. DC. Prodr. I. (1824) p. 146. Ledeb. Fl. Ross I. (1842) p. 121. Koch Syn. Fl. Germ. et Helv. ed III. (1857) p. 37. Regel Pl. Radd. I. (1861) p. 168. Wagner Illus. Deut. Fl. (1871) p. 48. Kom. Fl. Mansh. II. (1904) p. 378. Nakai Fl. Kor I. (1909) p. 54. 
A. Ovirensis, WuLF in JAcQuIN Icones plantarum rariorum I (1781) t. 125 et Collectanea ad botanicam I. (1786) p. 197. DC. Prodr. II. p. 146 .

A. stolonifera, Hornemann Hortus regius botanicus Hafniensis (1831-15) p. 618. DC. Prodr. II. 146.

Nom. Jap. Maruba-hatazao.

Hab.

Yeso: in oppido Yubetsu prov. Kitami (Hideo KoIdzumi).

Corea: in via ab oppido Musang ad oppidum Kapsan (V. Komarov n. 785). in monte Kum gang-san (U. FaURIE n. 607).

Distr. Germania, Austria, Russia, Sibiria, Kamtschatica et Manshuria.

7) Arabis senanensis, (Fr. et SAv.) Makino in Tokyo Bot. Mag. XXIV (1910) p. 224 et Somokudzusetsu Rev. ed. III. (1912) p. 880 P1. 724 .

A. Halleri, L. v. senanensis, Fr. et Sav. Enum. Pl. Jap. II. (1879) p. 279. Boiss. in Bull. Herb. Boiss. VII. (1899) p. 787. Matsum. Ind. Pl. Jap. II. 2. (1912) p. 147. NAKaI Veg. Isl. Quelp. (1914) p. 49. 1. 665 .

A. coronata, NakaI in Tokyo Bot. Mag. XXVIII (1914) p 302.

A. gemmifera, (Matsum.) Makino l.c.

A. lyrata, (non L.) Nakai Veg. Mt. Chirisan (1915) p. 33 n. 211.

A speciei precedente foliis majoribus et floribus majoribus differt. Ad finem florum caulis gemmas axillares quibus facile propagit agit.

Nom. Jap. Tsuru-tagarashi v. Hakusan-hatazao

Ilab.

Corea: in monte Paik-tu-san (TAMEzo Mori n. 69). in monte Chirisan (Takenoshin Nakai n. 743).

Quelpært: in herbidis Hallasan $2000 \mathrm{~m}$. (Favrie n. 1743). in orizetis Hongno (TAQUET n. 2625). monte Hallasan (TAKenoshin NAKaI n. 4793). in cinere Hallasan (TAQUE' n. 561). in silvis Sokpat $1000 \mathrm{~m}$. (TAQUET n. 4113). Hallasan $1800 \mathrm{~m}$. (TAQUET n. 2622), in silvis Mokatji $800 \mathrm{~m}$. (TAQUeT n. 559). in silvis Hallasan (TAQUeT n. 2613). in silvis (Taquet n. 538). in silvis Poptyyang (Taquet n. 5374).

Shikoku: monte Tsurugisan (GEN-ICHI KoIdzumi).

Hondo: Tahara prov. Bitchu (Zensuke Yoshino). in oppido Sakamoto-mura prov. Ise (Jintaro Umemura). in monte Ibukiyana (Gen-ichi Koldzumi). monte Hakusan prov. Kaga (Masataro Toyama, Jinzo Matsumura). monte Gassan prov. Uzen (Ryokichi Yatare, Gen-ichi KoIdzUMi). 
Yezo: Nemuro (KIngo Mi :ABË).

Planta endemica!

8) Arabis Stelleri, AU(x. DC. Regni Vegetabilis Systema Naturale II. (1821) p. 242.

var. japonica, (A. Gray) Fr. Schmidt Reis. in Amurlande und auf der Insel Sachalin (1868) p. 111. Fran. et Sav. Enum. Pl. Jap. I. (1875) p. 33 excl. syn. Honzodzufu. II. (1879) p. 278. Forbes et Hemsl. in Journ. Linn. Soc. XXIII (1886) p. 42. Palib. Consp. Fl. Kor. I. (1898) p. 26. Borss. in Bull. Herb. Boiss. VII (1899) p. 784. Yatabe Icon I. t. 1. Matsum. Ind. Pl. Jap. II. 2 (1912). p. 148.

A. Stelleri, (non DC.) MiQ. Prol. Fl. Jap. (1866-7) p. 4. Nakai Fl. Kor. I. (1909) p. 54. KoIdz. Pl. Sachal. p. 72.

A. japonica, A. Gray on the Botany of Japan p. 381. Mrg. Prol. Fl. Jap. (1866-7) p. 363. TAKEDA in Journ. Linn. Soc. XIII (1914) p. 452. Miyabe et Miyake Fl. Sachal. (1915) p. 39.

A. japonica, Regel et Herder in Gartenfl. (1863) p. 308 t. 414.

A. alpina, L. var. ? japonica, A Gray Perry's Exped. (1857) p. 307.

Nom. Jap. Ezo-hama-hatazao.

$\mathrm{Hab}$.

Sachalin : Toreipachi et Korsak of (Genji Nakahara).

Yeso: Sapporo (Kingo Miyabe) sine loco speciali (Boemmer). Yubutsu prov. Iburi. (Kingo Miyabe).

Hondo: in littore Aikawa insulæ Sado (Ryokichi Yatabe). Honmura insulæ Niijima prov. Idzu (Saburo Oкuвo). Hamanoshiba prov. Kadzusa (?).

var. stenocarpa, Fr. et SAv. Enum. Pl. Jap. II. (1879) p. 278. Borss. in Bull. Herb. Boiss. VII. (1899) p. 784.

Nom. Jap. Hama-hatazao.

Hab.

Yeso: Zenibako prov. Shiribeshi (Y. ToкuBuchI). ibidem (Jinzo Matsumura). sine loco speciali (Ryokichi Yatabe).

Hondo: inter Sakata et Honjo prov. Uzen (Ryokichi Yatabe). sine loco speciali prov. Echigo (Ryokichi Yatabe). in littore Nishi-iwase prov, Etchu (Jinzo Matsumura).

Quelpært: Kantoku (TAKenoshin NAKaI n. 14.36). Hallasan 1500 m. ('TAQUET n. 5389).

var. macrocarpa, NAKAI var. nov.

Folia ovata amplexicaulia. Siliqua subtortuosa usque $7 \mathrm{~cm}$. longa $1.5 \mathrm{~mm}$. lata apice stylum attenuata.

Nom. Jap. Takeshima-hama-hatazao. 
Hab.

Corea : in littore insulæ Ooryöngto (Takenoshin NakaI n. 4309). Plantæ endemicæ !

9) Arabis nipponica, Borss. in Bull. Herb. Boiss. VII (1899) p. 785. Matsum Ind. Pl. Jap. II. 2. (1912) p. 149.

A. hirsuta v. nipponica, Fr. et SAv. Enum. P1. Jap. I. (1875) p. 34.

A. sagittata, (non DC.) Matsum. Ind. P1. Jap. II. 2. (1912) p. 148.

A. sagittata, v. nipponica, (Fr. et SAv.) MAtsum. 1.c. p. 149.

A. hirsuta, (non Scop.) Maxim. Prim. Fl. Amur. (1859) p. 44. Regel Tent. Fl. Uss. n. 63. Forbes et Hemsl. in Journ. Linn. Soc. XXIII (1886) p. 42. Korsch. in Act. Hort. Petrop. XII. p. 307. Pal.ib. Cons. Fl. Kor. I. (1898) p. 26. Kom. Fl. Mansh. II. (1904) p. 376 NaKaI Fl. Kor. I. (1909) p. 54. II. (1911) p. 440. MaKino in Somokudzusetsu Rev. Edit. (1912) p. 889. pl. 734.

Icon. Honzozuhu Vol. XXXIX fol. 16. dextr.

Nom. Jap. Yama-hatazao.

All Asiatic Arabis hirsuta is A. nipponica. It becomes always larger in size and the cauline leaves are more strongly amplexicaulis. Its stem is covered by the stellate hairs (sometimes intermixed with the simple one) while the typical $A$. hirsuta has simple hairs. The outer feature of an weak and poor specimen resembles to A. hirsuta, but the wider cauline leaves and the stellate hairs on the stem kecp always their invariability.

Hab.

Corea: monte Kum-gang-san (Takenoshin NaKaI n. 5449). Sea Kong-go (Takenoshin Nakai n. 5450). Panja montium Chirisan (Takenoshin Nakai n: 753). monte Chirisan (Takenoshin Nakai $n$. 4788-9), in colle Ouensan (Takenoshin Nakal n. 4791). Kang-gei (Mrlus n. 321). insula Takigi-shima circa Sei-ko-sin (TAKenoshin NAKAI n. 4791). in rupibus secus mare Ouensan (FAURIE n. 123) in petrosis Seoul (Faurie n. 605). in arce Pouk-han $1000 \mathrm{~m}$. (Faurie n. 131).

Quelpært: monte Hallasan (TAKenoshin NAKAI n. 961). insula parva Pi-yang-to (TAKenoshin NAKaI n. 4790). circa Sai-shu (TAKENoshin NAKAI n. 969). in herbidis (FAURIE n. 1744). in agris (FAURIE n. 5390). in agris Hongno (TAQUeT n. 552). in agris Polmongi (TAQUeT n. 2607).

Austro-Ussuri : Vallis Liu-cze-chesa (V. Komarov n. 784).

Hondo: monte Harunasan prov. Kozuke (Saburo Orubo). Aidzu prov. Iwashiro (Jinzo Matsumura) Tokyo prov. Musashi (Jinzo 
Matsumura). inter Oiwake et Komoro prov. Shinano (Jinzo Matsumura). Aomori prov. Mutsu (Nobutaro Kinashi). Sanmata prov. Tamba (Kei Takenouchi). Asamayama prov. Shinano (Jinzo Matsumira). Nikko prov. Shimotsuke (Komajiro Sawada). monte Togakushi prov. Shinano (Jinzo Matsumura). Totaru prov. Idzu (Jinzo Matsumura). Yokohama prov. Musashi (Jinzo Matsumura).

Kuril : insula Urupp (Tasaku Kitahara).

Kiusiu: Nittanohara prov. Bungo (Hamada).

Distr. China?

10) Arabis yokoscensis, Fr. et SAv. Enum. Pl. Jap. I. (1875) p. 34. II. (1879) p. 279. Matsum. Ind. Pl. Jap. II. 2. (1912) p. 149.

Hab.

Hondo: Yokosuka prov. Sagami.

Planta endemica!

11) Arabis Boissieuana, NakaI. sp. nov.

A. amplexicaulis, (non Edgeworth) Fran. et Sav. Enum. Pl. Jap. I. (1875) p. 33. Matsum. Ind. Pl. Jap. II. 2. (1912) p. 147.

A. amplexicaulis var. japonica, Borss. in Bull. Herb. Boiss. VII. (1899) p. 786.

A. Gerardi, (non Bess.) MrQ. Prol. Fl. Jap. p. 4.

This species is nearest to Arabis Fauriei and $A$. serrata, still differs from them by having not cæspitose stem, more remote serrations and much more elongate and straight siliques. This was also commixed with Arabis amplexicaulis and $A$. Gerardi, but the former is a Himalayan plant having simple undivided hairs on the stem and the latter is an European one with reticulate sceds. I have named after the late H. DE Borssieu who first noticed the difference between this and Arabis amplexicaulis and distinguished this as a variety of the latter.

Radix perennis interdum columnalis $\mathrm{v}$. cum sobolibus ramosa. Caulis pilis stellatis $v$. bifurcatis adpressis pilosus $16-45 \mathrm{~cm}$. altus teres viridis. Folia radicalia spathulata in petiolem alato-attenuata utrinque sparsim sed supra crebrius stellulato-pilosa grosse paucique dentata acutiuscula. Folia caulina amplexicaulia oblonga v. elongato-elliptica grosse æqualiter $\mathrm{v}$. inæqualiter serrata apice mucronata, basi auriculata v. subsagittata. Racemus polyanthus ebracteatus. Pedicelli glabri usque $15 \mathrm{~cm}$. longi. Sepala glabra viridia basi leviter saccata $5 \mathrm{~mm}$. longa 1-1.5 mm. lata margine albida. Petala alba 7-9 $\mathrm{mm}$. longa spathulata. Styli $1 \mathrm{~mm}$. longi. Stigma punctatum. Ovarium glabrum v. stellulato-pilosum. 
Nom. Jap. Iwa-hatazao.

Mab.

Hondo: monte Hondojisan prov. Uzen (SabURo OKuBo) monte Nyoho prov. Shimotsuke (GeN-IChI KoIdzumi). Nikko prov. Shimotsuke (Yoshisada YABE$_{\text {A }}$ ). monte Adzumasan prov. Iwashiro (Genji NAKAHARA). Sine loco special prov. Yamato ( $T$. TADA). in monte Zao 藏王山 prov. Iwaki (HIRoshi NAMBU).

Shikoku: monte Yahazuyama prov. Tosa (Ryokichi Yatabe).

var. nikoensis, NAKAI. var. nov.

Caulis solitarius (an semper?). Folia margine undulata v. brevissime serrata oblonga $\mathrm{v}$. lineari-oblonga $v$. lanceolata, caulina basi amplexicaulia.

Nom. Jap. Nikko-hatazao.

Hab.

Hondo: in summo montis Okujirane, Nikko (GeN-IChi KoIDzumi, Hisayoshi Takeda). monte Shirane, Nikko (Komajiro Sawada). Misawa, Nikko (Jinzo Matsumura).

var. shikokiana, NAKAI. var. nov.

A typo differt caule glabriore, foliis conspicue grosse serratis ct glabris, floribus majoribus.

Radix perennis lignosa. Caulis ascendens cæspitem 2-3 pilis adpressissimis bifurcatis sparsissime pilosus $v$. fere glaber, usque $40 \mathrm{~cm}$. altus. Folia radicalia elongato-spathulata grosse sinuato-dentata apice obtusa utrinque glabra, margine pilis bifurcatis pilosa. Folia caulina ablonga basi auriculato-amplexicaulia præter marginem glabra, margine grosse acute serrata, apice acuta v. acuminata. Racemns elongatus ebracteatus glaber polyanthus. Pedicelli floriferi usque $1.5 \mathrm{~cm}$. glaberrimi. Sepala glabra $4.5 \mathrm{~mm}$. longa $1 \mathrm{~mm}$. lata. Petala alba obovato-spathulata. 9-10 $\mathrm{mm}$. longa $4 \mathrm{~mm}$. lata. Ovarium glaberrimum. Styli $1 \mathrm{~mm}$. longi. Siliqua erecta subtorulosa compressa $7.5-9 \mathrm{~cm}$. longa $1.2-1.5 \mathrm{~mm}$. lata. Semina apice alata atro-fusca sub lente reticulato-punctata.

Nom. Jap. Shikoku-hatazao.

Hab.

Shikoku : in Nanogawamura prov. Tosa (WATANABE).

Planta endemica!

12) Arabis takesimana, Narar. sp. nov.

Biennis. Caulis cum inflorescentia $19-36 \mathrm{~cm}$. altus glaberrimus. Folia radicalia rosulata spathulata in petiolo attenuata $v$. decurrentia integra $v$. breve dentata, supra sparsissime stellulato-pilosa, infra 
præter costas stellulato-pilosas glaberrima 1-7 cm. longa. Petioli basi margine pilis simplicibus sed superne stellulato-pilosi. Folia caulina lanceolata $v$. ovato-oblonga inferiora remote breveque dentata et sparsissime stellulato-pilosa, superiora integerrima et glaberrima apice obtusa, basi amplexicaulia v. sagittato-amplexicaulia. Inflorescentia racemosa ebracteata glaberrima polyantha. Pedicelli glaberrimi graciles inferiores longiores usque $1.5 \mathrm{~cm}$. longi. Sepala viridia $3-3.5 \mathrm{~mm}$. longa $1 \mathrm{~mm}$. lata obtusa basi leviter saccata. Petala alba obovatospathulata 7-8 mm. longa $3 \mathrm{~mm}$. lata. Stamina brevia $3 \mathrm{~mm}$. longa $5 \mathrm{~mm}$. longa. Antheræ flavæ. Ovarium glaberrimum. Siliqua maturitate patentia $\mathrm{v}$. pendula $5-8.5 \mathrm{~cm}$. longa $1-1.3 \mathrm{~mm}$. lata. Semina 1 $\mathrm{mm}$. lata $1.5 \mathrm{~mm}$. longa atro-fusca basi alata.

Nom. Jap. Takeshima-hatazao.

Hab. in insula Ooryongto: in silvis (Takenoshin NakaI $n$. 4307-8, 4305, 4302, TsuTomu Ishidoya 50-51).

\section{f. lancea, NAKAI.}

Folia radicalia lineari-oblanceolata, caulina lineari-lanceolata.

Hab. in insula Ooryongtō: in silvis Rarikol, rara (Takenoshin NAKAI n. 4304).

\section{f. latifolia, NAKAI.}

Folia radicalia obovata, caulina inferiora fere rotundata superiora ovata v. lanceolata.

Hab. in insula Ooryongto: in silvis Obokdong, rara ('TAKENoshin Nakai n. 4303).

13) Arabis pseudo-auriculata, Borss. in Bull. Herb. Boiss. VII (1899) p. 787 Matsum. Ind. P1. Jap. II. 2. (1912) p. 148.

Nom. Jap. Ezo-yama-hatazao.

Hab.

Yeso: Maruyama in Sapporo prov. Ishikari (Hisayoshi Takeda) Iwanai prov. Shiribeshi (FAURIE) insula Shakotan (FAURIE).

Planta endemica!

14) Arabis glauca, Boiss. Bull. Herb. Boiss. VII (1899) p. 786. Matsum. Ind. Pl. Jap. II. 2 (1912) p. 147.

Nom. Jap. Ezo-iwa-hatazao.

Hab.

Yeso: Sapporo prov. Ishikari (FAurie). Sozan (FAURIE).

Planta endemica!

15) Arabis Fauriei, Boiss. in Bull. Herb. Boiss. VII (1899) p. 787. Matsum. Ind. Pl. Jap. II. p. 147.

A. Iwatensis, Makino in Tokyo Bot. Mag. XVIII (1904) p. 113, 
Matsum. 1.c. p. 148.

Nom. Jap. Iwate-hatazao.

Affinis $A$. serratæ sed exqua foliis non sinuato-serratis, glabrioribus, siliquis angustioribus.

Hab.

Hondo: monte Iwatesan (Kadzuo Koidzumi, Shozo Yajima, Genji NAKAHARA). Zaimoku-one montis Yatsugatake prov. Kai (Hisayoshi TAKEDA). monte Shirouma prov. Etchu (Hisayoshi Takena).

var. grandiflora, NAKAI var. nov.

Radix perennis sæpe ramosa. Planta cum soboles propagit cæspitosa. Caulis cum inflorescentia usque $12 \mathrm{~cm}$. altus basi foliis emortuis imbricatis, cum infructescentia usque $25 \mathrm{~cm}$. altus sæpe purpureus. Folia radicalia spathulata usque $4 \mathrm{~cm}$. longa viridia $v$. apice purpurascentia basi attenuata apice obtusa, margine grosse crenatodentata. Pagina utrinque stellulato sparsim pilosa. Folia caulina ovata v. elliptica $\mathbf{v}$. oblonga sessilia amplexicaulia grosse breve dentata utrinque stellulato-pilosa. Inflorescentia racemosa polyantha. Sepala viridia margine alba $4 \mathrm{~mm}$. longa $2 \mathrm{~mm}$. lata apice acuta v. obtusiuscula basi saccata. Petala alba obovata spathulata 9-10 mm. longa 3-4 mm. lata. Stamina brevia $5 \mathrm{~mm}$. longa, elongata $6 \mathrm{~mm}$. longa. Ovarium glaberrimum. Styli $1 \mathrm{~mm}$. longi. Stigma bilobum papillosum. Siliqua suberecta $2-6 \mathrm{~cm}$. longa $1.5-2.3 \mathrm{~mm}$. lata apice stylum persistentem attenuata pedicello $3-15 \mathrm{~mm}$. longo. Semina compressa lævia castanea alata $1 \mathrm{~mm}$. longa fere rotunda.

Nom. Jap. Ume-hatazao.

$\mathrm{Hab}$.

Hondo: in alpinis Shirouma prov. Etchu. (Shinsuke Kodama). in herbidis humidis alpini Yarigatake prov. Etchu (Matsuwaka Kishida, Hisayoshi Takeda, Genichi Koidzumi).

Planta endemica!

16) Arabis serrata, Fran. et Sav. Enum. Pl. Jap. I. (1875) p. 33. II. (1879) p. 278. Borss. in Bull. Herb. Boiss. VII (1899) p. 787. Matsum. Ind. P1. Jap. II. 2. (1912). p. 149.

A. amplexicaulis, v. serrata, Makino in Tokyo Bot. Mag. XXIII. p. 16.

A. hallaisanensis, NakaI in Fedde Rep. XIII (1914) p. 271.

Nom. Jap. Fuji-hatazao.

Cum multis intermediis formis interpositis A. hallaisanensis sensim in A. serratam transit.

Hab. 
Hondo: monte Fuji (Jinzo Matsumura, Takenoshin Nakai). Quelpært: Hallasan (Tsutomu Ishidoya, Faurie, Taquet). Planta endemica!

17) Arabis Kishidai, NAKar. sp. nov.

This is near to $A$. Boissieuana v. nikoensis which is an extreme form of $A$. Boissieuana, but differs from it by having more cæspitose stems and more stellulate-haired leaves. By its cæspitose habit it somewhat resembles to $A$. Fauriei, but is readily distinguishable by its narrower cauline leaves which are entire or inconspicuously serrulate in their margines and more slenderer straight siliques as well as more elongate racemes.

Radix perennis. Caudex breve ramosa et incrassata lignosa foliis emortuis imbricatis dense vestita. Caulis robustus $6-22 \mathrm{~cm}$. altus subglaber v. stellulatus. Folia radicalia spathulata margine repanda utrinque minute sed crebri stellulato-pilosa, usque $3 \mathrm{~cm}$. longa, caulina oblonga $\mathbf{v}$. elliptica $\mathbf{v}$. ovato-oblonga integra $\mathbf{v}$. indistincte serrulata 1.5-2 cm. longa 5-8 mm. lata amplexicaulia. Raccmus suls anthesin brevis corymbosus, sed in fructu elongata. Sepala basi saccata 2.5 $\mathrm{mm}$. longa. Petala alba 4-5 $\mathrm{mm}$. longa obovata. Siliqua angusta apice stylos attenuata. Stigma punctatum leviter emarginatum.

Nom. Jap. Shozu-hatazao.

Hab.

Hondo: in monte Shozugatake 清水岳 prov Eitchu (MatsuwaKa KIsfida) in Tengu-bishi montis Yarigatake prov. Etchu (Matsuwaka Kishida). Planta endemica!

18) Arabis Tanakana, Makino in Tokyo Bot. Mag. XVII (1003) p. 160. Matsum. Ind. Pl. Jap II. 2. (1912) p. 149. Nom. Jap. Miyama-hatazao. Hab.

Hondo: monte Yarigatake prov. Etchu (Körchi Tanaka). monte Ainotake montium Shirane prov. Kai (TôIchIRô AsaI). Planta edemica!

19) Arabis Kawasakiana, MAKINo in Tokyo Bot. Mag. XXVII (1913) p. 24.

A. petræa var? Makino in Tokyo Bot. Mag. XI (1897) p. 157.

A. petræa var. nipponica, Makino Somokudzusetsu Rev. Edit. (1912) Vol. XII. p. 10 t. 11.

Nom. Jap. Tachi-suzushiroso. Hab. 
Hondo: in littore maris Yokkaichi prov. Ise.

Planta endemica!

20) Stenophragma Thalianum, (L.) CELAK. in Arch. Naturw. Landesd. Boehm. III. (1875) p. 445. Prantl in Nat. Pflanzenf. III. 2. (1890) p. 192. Wats. Syn. Fl. I. i. (1895) p. 140.

S. Thaliana, Celak. in Oest. Bot. Zeits. XXVII (1877) p. 177. Britton and Brown Illus. Fl. Northern United States and Canada II. p. 146.

Sisymbrium Thalianum, Gay. in Ann. Sci. Nat. Ser. I. VII. (1826) p. 399. et in Gaud. Fl. Helv. IV. p. 348.

Arabis Thaliana, L. Sp. Pl. (1753) p. 665. DC. Prodr. I. p. 144. Sowerby Engl. Bot. I. (1873) t. CXV. Wagner Illus. Deut. Fl. p. 49. f. 75. Fran. et Sav. Enum. Pl. Jap. II. (1879) p. 280. Borss. in Bull. Herb. Boiss. VII. (1899) p. 789. Matsum. in Tokyo Bot. Mag. XIII. (1899) p. 59. Ind. Pl. Jap. II: 2. (1912) p. 149. Makino in Tokyo Iot. Mag. XXVII (1913) p. 26.

A. pubicalyx, Mro. Prol. Fl. Jap. (1866-7) p. 4. Fr. et Sav. Enum. Pl. Jap. I. (1875) p. 34.

A. pubicalyx v. soyensis, Borss. in Bull. Herb. Boiss. VII. (1899) p. 788.

A. Thaliana v. pubicalyx, Makino Somokudzusetsu Rev. ed. (1912) Vol. XII. p. 11. t. 12.

Nom. Jap. Shiro-inu-nadzuna.

$\mathrm{Hab}$.

Hondo: Fukumoto prov. Bizen (Gen-IChi Koidzumi). Öuchimura districtu Yoshikigun prov. Suō (Jiuro NikaI n. 243). Kawarabayashi prov. Tamba (Kei Takenouchi).

Corea: Riri (Takenoshin Nakai n. 1151), inter Seiyu et Chojo (Takenoshin Nakai n. 4792).

China: Kong-san-mun circa Hang-chao prov. Che-Kiang (Arsuji Honda). in montibus Che-kiang (Chang). Soo-choo prov. Kiang-su (Shinzo OKA).

Distr. Europa et America bor.

\section{ERRATA.}

\begin{tabular}{|c|c|c|c|c|c|c|c|}
\hline P. & 217 & line. & 32 & $10 \mathrm{co}$ & de-currertibus & lege & decurr \\
\hline$"$ & 222 & " & 8 & "' & S. & ", & et ARN. \\
\hline & $2: 26$ & $"$ & $\begin{array}{l}24 \\
28\end{array}$ & $"$ & $\begin{array}{l}\text { intcgcrima } \\
\text { chæroyhylloides }\end{array}$ & " & $\begin{array}{l}\text { integerrima. } \\
\text { chærophylloides. }\end{array}$ \\
\hline & 227 & $"$ & 29 & & Hob. & " & Hab. \\
\hline & 231 & & 23 & excl. & pargine. & & \\
\hline & & $"$ & & IOQ & & & \\
\hline
\end{tabular}

